\title{
A new spinning technique for hollow fiber ultrafiltration membranes
}

\author{
I.M. Wienk, H.A. Teunis, Th v.d. Boomgaard and C.A. Smolders \\ University of Twente, P.O. Box 217, 7500 AE Enschede (The Netherlands)
}

(Received September 7, 1992; accepted in revised form November 2, 1992)

\begin{abstract}
A new spinning technique for hollow fiber membranes with a densified outer toplayer has been developed in our laboratory. This technique makes use of a new type of spinneret having three concentric orifices. Apart from polymer solution and bore liquid as applied in classical spinnerets a third liquid can be pumped through an outer layer. After a certain contact time with the third liquid the nascent hollow fiber membrane meets the coagulation bath. This spinning technique has been applied to produce hollow fiber ultrafiltration membranes. The presence of poly-vinylpyrrolidone in the polymer solution is shown to be essential to induce a porous toplayer. Membranes having a waterflux of $275 \mathrm{~L}-\mathrm{m}^{-2}-\mathrm{hr}^{-1}-\mathrm{bar}^{-1}$ and BSA retention of $97 \%$ have been spun. Although more research has to be done the first results seem to be very promising.
\end{abstract}

Keywords: membrane formation; spinning; ultrafiltration; hollow fiber membranes

\section{Theoretical introduction}

In practical applications of ultrafiltration membranes permeabilities are usually low due to low surface porosities and severe fouling tendencies. Several researchers [1] have shown that fouling can be reduced by applying hydrophilic membranes giving a significant decrease in adsorption of proteins at the membrane surface. Roesink [2] was able to produce phase inversion microfiltration membranes with a hydrophilic character by adding polyvinylpyrrolidone (PVP) to the polymer solution. In the present investigation hollow fiber ultrafiltration membranes are made from a so-

Correspondence to: I.M. Wienk, University of Twente, P.O. Box 217, 7500 AE Enschede, The Netherlands. lution containing poly-ethersulfone (PES) and PVP.

Membrane formation by immersion precipitation for systems containing PES and PVP has been studied thoroughly by Boom [3]. $\mathrm{He}$ showed that the introduction of PVP to the membrane forming system has a profound influence on the morphology of the membrane: macrovoid formation can be suppressed and pores become very well interconnected. PES and PVP are miscible but upon immersion in a waterbath the PVP wants to move towards the water. Boom found that demixing of these systems is mainly determined by the phase separation of the two polymers. This implies diffusion of PES and PVP relative to each other which is a slow process compared to solvent and non-solvent exchange. Therefore the composi- 
tion of the polymer solution will become highly unstable without nucleation of a polymer lean phase taking place. In these systems spinodal demixing occurs and a bi-continuous structure is obtained. Boom proposed that the interconnectivity of the pores is a result of spinodal demixing of the polymer solution.

Cabasso et al. [4] found that PVP increases pore size and that addition of PVP is not suitable in preparing membranes with a dense toplayer, like applied for gas separation or pervaporation. Usually membranes made with PVP in the solution have no distinct toplayer but pore sizes increase gradually from one side to the other.

It is anticipated that the permeability of PES/PVP ultrafiltration membranes should increase substantially when the structure of these membranes consists of a thin selective toplayer and a very porous sublayer. For gas separation membranes starting with a solution of one polymer, it is known [5] that membranes with thin, dense toplayers can be formed if two successive coagulation baths are used. The nonsolvent liquid used as the first coagulation medium was chosen in such a way that the solvent flux out of the polymer solution is high, while the inflow of this nonsolvent is very low. Therefore the polymer concentration at the interface is increased. In the second bath phase separation occurs. Due to the high interfacial polymer concentration a thin gas-tight toplayer is formed.

With the new type of spinneret that has recently been developed [6] in our laboratory the dual bath system can be applied in the continuous spinning process for making ultrafiltration membranes. The triplet spinneret has three concentric orifices (Fig. 1). The polymer solution is pumped through the middle opening. The inner orifice is used for the internal coagulation bath or bore liquid. This medium determines the pore structure at the inner (bore) surface and prevents the fiber from collapse during
First external coagulation medium
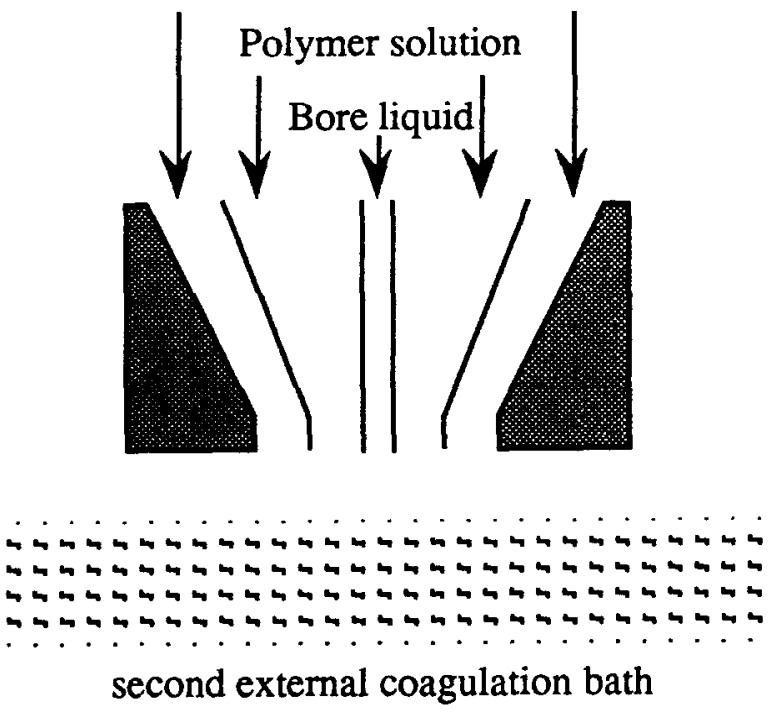

Fig. 1. Triple layer spinneret, schematic drawing. The streams are pumped through the three concentric openings simultaneously. After a certain contact time they enter the second bath where the outer liquid layer is exchanged.

spinning. The first external coagulation medium is pumped through the outer opening. The three liquid streams are extruded from the spinneret and after a certain contact time they enter a waterbath. In this second external coagulation bath the first coagulation medium is replaced by water and coagulation proceeds quickly.

\section{Materials and methods}

\section{Materials}

Poly-ethersulfone (PES) was purchased from ICI (Victrex 5200P). Using GPC the weight average molecular weight was determined at $44,000 \mathrm{~g} / \mathrm{mole}$. Two kinds of poly-vinylpyrrolidone (PVP) from Janssen Chimica were used, $\mathrm{K} 90\left(M_{\mathrm{w}} 507,000 \mathrm{~g} / \mathrm{mole}\right)$ and $\mathrm{K} 30$ $\left(M_{\mathrm{w}} 18,000 \mathrm{~g} / \mathrm{mole}\right)$. The solvent 1-methyl-2pyrrolidone (NMP) was purchased from Merck (synthetic grade). Nonsolvents were water, 2- 
propanol, 1-pentanol and dehydrated glycerol. The last three were purchased from Merck (analysis grade).

\section{The spinning process}

PES and PVP were dissolved in NMP and water was added. Two different polymer casting solutions were used:

Solution A: $\quad$ PES/PVP(K90)/PVP(K30)/ water/NMP 20/5/5/5/65 wt.\%

Solution B: $\quad$ PES/PVP(K30)/water/ NMP $20 / 10 / 5 / 65$ wt. $\%$

Filtered polymer solutions and liquids used as first coagulation medium were degassed before use. Spinning temperature of the polymer solution was $50^{\circ} \mathrm{C}$, coagulation baths were used at $25^{\circ} \mathrm{C}$. After spinning and coagulation the fibers were rinsed with hot water for two days. Following Roesink [2], excess PVP was removed by contacting the fibers with a $4000 \mathrm{ppm}$ sodium hypochlorite solution for $48 \mathrm{hr}$. After rinsing for one more day the fibers were put in a waterbath containing $10 \mathrm{wt} . \%$ glycerol for two days and finally dried at room temperature.

\section{Characterization methods}

Membrane morphology was studied using a scanning electron microscope (JEOL, JSM T220A). Water for flux measurements was prefiltered by reverse osmosis. Retentions were measured using either a BSA solution or a PEG solution. BSA $\left(M_{\mathrm{w}} 65,000\right)$ was purchased from Sigma Chemical company and dissolved in a phosphate buffer solution of $\mathrm{pH} 7.4$, containing $0.1 M \mathrm{NaCl}$. Poly (ethylene glycol), PEG $\left(M_{\mathrm{w}}\right.$ 40,000 ) was purchased from Serva. Flux and retention measurements were carried out in a crossflow filtration setup. Small modules were made containing five fibers with internal diameter of $0.8 \mathrm{~mm}$ and external diameter of 1.3 $\mathrm{mm}$ and a length of $20 \mathrm{~cm}$. The applied pressure difference was 2 bars and after $30 \mathrm{~min}$ condi- tioning time the waterflux was almost constant. BSA and PEG concentrations were determined spectrophotometrically.

\section{Light transmission measurements for} determination of delay times

For flat membranes delay times were measured using a setup in which a light detector is placed below a coagulation bath and a light source above [7]. The output of the light detector is converted into a recorder signal. The delay time is defined as the time between immersion of the polymer film and the beginning of turbidity in the nascent membrane. The turbidity indicates the onset of precipitation and is detected by a drop in light transmittance through the polymer film.

\section{Results}

\section{Light transmission}

For polymer solution A, immersed in a number of liquids to be used as the first coagulation bath, delay times have been measured. The coagulation baths are non-solvents for PES but solvents for PVP. The transmittance decrease in time is depicted in Fig. 2. In all cases delay times were zero, meaning that demixing of the polymer films starts instantaneously after immersion. However the velocity of decrease in transmittance was different for all nonsolvents and it increased in the following order:

glycerol $<$ (50 wt.\% 2-propanol in glycerol ) $<1$ pentanol $<2$-propanol $<(20$ wt.\% NMP in water) <water.

\section{Spinning of ultrafiltration membranes}

Ultrafiltration membranes have been spun using the triple layer spinneret. For polymer solution A the nonsolvents, or mixtures of non- 


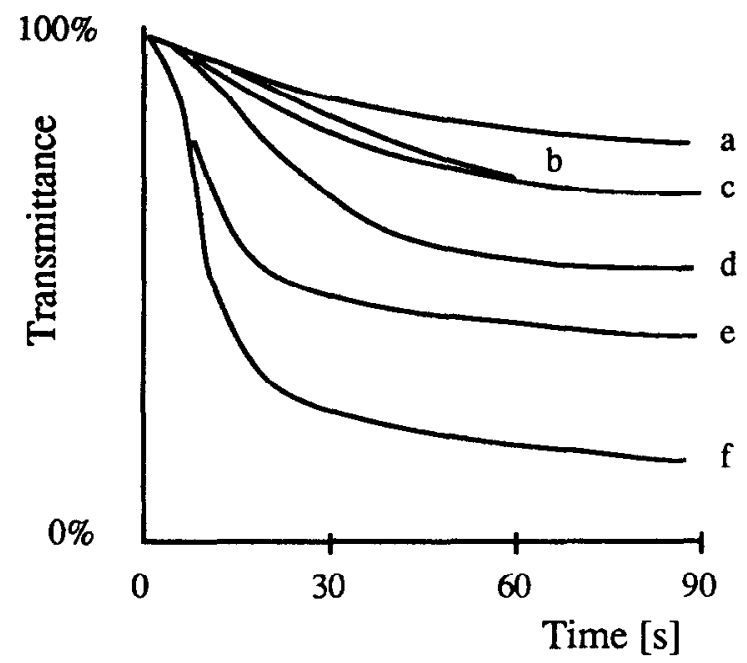

Fig. 2. Transmittance decrease in time for films of polymer solution A immersed in a number of liquids: (a) glycerol, (b) 50 wt.\%-propanol in glycerol, (c) 1-pentanol, (d) 2 propanol, (e) $20 \mathrm{wt} . \%$ NMP in water, (f) water.

solvents as given in Table 1 have been used as a first coagulation medium. Polymer solution, bore liquid and first coagulation medium were pumped through the spinneret. After $0.5 \mathrm{sec}$ these streams entered a waterbath of $20^{\circ} \mathrm{C}$ were the first nonsolvent was exchanged with water. We tried to use water for both the first and the second bath but the fibers broke during spinning and could not be measured.

Waterfluxes and retentions for BSA have been measured for all fibers; values are listed in Table 1. For comparison an experiment with a conventional spinneret (tube-in-orifice) is added. In this case the first coagulation medium is air at ambient temperature and humidity conditions after which the fiber enters the waterbath.

In Table 1 it can be seen that the use of 1pentanol as a first coagulation medium results in a substantially higher flux compared to the flux in case an airgap is used. If glycerol is used waterflux is somewhat higher than with an airgap but retention is much lower.

The structure of the toplayer has been studied using SEM. Photographs of two membranes, prepared with 1-pentanol and air as the first coagulation medium are shown in Fig. 3. In the case of 1-pentanol the transition from toplayer to porous sublayer is very clear, toplayer thickness was $0.5 \mu \mathrm{m}$ in this case. Toplayer thickness of the membrane fiber made with the tube-in orifice spinneret is $1 \mu \mathrm{m}$ and pore sizes in the sublayer are much smaller. The membrane structure in the toplayer consists of

\section{TABLE 1}

Water flux and BSA retention for hollow fibers spun with different first external coagulation media. Polymer solution $\Lambda$ was used. The contact time of the polymer solution with the first coagulation medium was 0.5 sec. In all cases the second external coagulation bath was a waterbath of $20^{\circ} \mathrm{C}$. The bore liquid was $80 \% \mathrm{NMP}$ in water

\begin{tabular}{llr}
\hline First external coagulation medium & $\begin{array}{l}\text { Waterflux } \\
\left(\mathrm{L}-\mathrm{m}^{-2}-\mathrm{hr}^{-1}-\mathrm{bar}^{-1}\right)\end{array}$ & $\begin{array}{l}\text { BSA retention } \\
(\%)\end{array}$ \\
\hline Glycerol & 163 & 84 \\
2-Propanol in glycerol, 50 wt.\% & 110 & 63 \\
1-Pentanol & 275 & 97 \\
2-Propanol & 140 & 91 \\
NMP in water, 20 wt.\% & 148 & 94 \\
Airc & 137 & 100 \\
\hline
\end{tabular}

aDeviation from mean flux measurements is within $10 \%$.

${ }^{b}$ BSA concentration in the feed solution was $0.1 \mathrm{wt}$. \%. Deviation from mean for retention measurements is within $2 \%$.

'Spun with normal tube-in-orifice spinneret; instead of a first external coagulation medium the spinning solution passed an air gap before it entered the waterbath. 

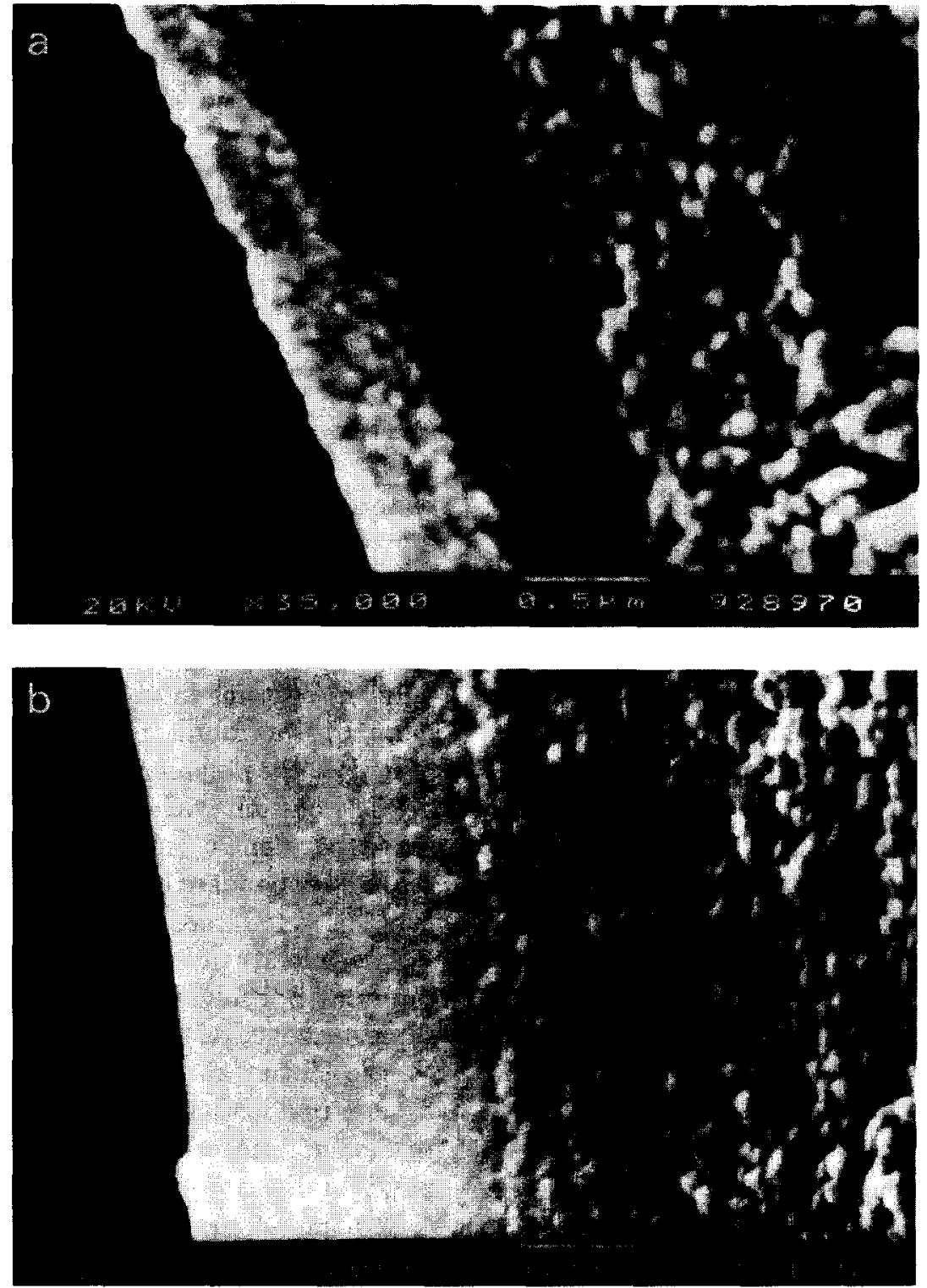

Fig. 3. Toplayer of hollow fiber membranes spun with two different first coagulation media, 1-pentanol (a) and air (b). The second coagulation medium was water.

polymer spheres. The formation mechanism of this nodular structure will be discussed in a forthcoming paper.

In a different set of experiments polymer solution $B$ was used also to investigate the influence of the waterbath temperature on water- flux and retention. The results in Table 2 show that waterflux is independent of the temperature of the second bath while retention decreases with increasing temperature.

For other ultrafiltration membranes that have a selective toplayer at the inside of the fi- 
TABLE 2

Influence of the temperature of the waterbath, being the second external coagulation bath, on the waterflux and the PEG retention. Polymer solution $B$ was used. The first external coagulation bath was $10 \mathrm{wt} . \%$ NMP in water, the contact time was $0.5 \mathrm{sec}$. The bore liquid was $80 \mathrm{wt} . \% \mathrm{NMP}$ in water

\begin{tabular}{lll}
\hline $\begin{array}{l}\text { Temperature of waterbath } \\
\left({ }^{\circ} \mathrm{C}\right)\end{array}$ & $\begin{array}{l}\text { Waterflux } \\
\left(\mathrm{I}_{\mathbf{a}}-\mathrm{m}^{-2}-\mathrm{hr}^{-1}-\mathrm{bar}^{-1}\right)\end{array}$ & $\begin{array}{l}\text { PEG retention } \\
(\%)\end{array}$ \\
\hline 23 & 57 & 99 \\
43 & 52 & 92 \\
60 & 66 & 75 \\
\hline
\end{tabular}

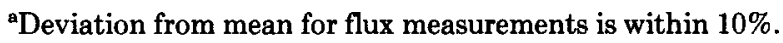

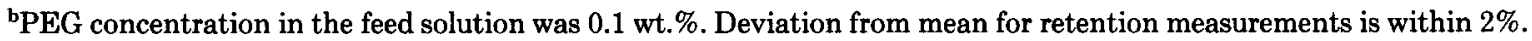

ber the triple layer spinneret can be used to obtain outer surfaces with large pores. In Table 3 pore sizes of the outer surface, as determined from SEM pictures are compared for different coagulation baths. If the triple layer spinneret is used as in the first two experiments of Table 3 , pore sizes are considerably larger than in case of the normal tube-in-orifice spinneret. From the first two experiments of Table 3 it can be seen that in this case the temperature of the waterbath influences the pore size at the surface. At a higher temperature of the second bath pore size is larger. The three membranes of Table 3 are all ultrafiltration membranes with the selective toplayer at the bore side. Therefore

\section{TABLE 3}

Mean pore size at the outer surface of ultrafiltration membranes with the selective toplayer at the inside of the fiber. Polymer solution A was used. In all cases the nascent membrane entered the sccond external coagulation bath (water) after $0.5 \mathrm{sec}$. The internal coagulation bath was $20 \mathrm{wt} . \%$ NMP in water

\begin{tabular}{lll}
\hline $\begin{array}{l}\text { First coagulation } \\
\text { medium }\end{array}$ & $\begin{array}{l}\text { Temperature of } \\
\text { second bath } \\
\left({ }^{\circ} \mathrm{C}\right)\end{array}$ & $\begin{array}{l}\text { Pore size } \\
(\mu \mathrm{m})\end{array}$ \\
\hline NMP in water, 80 wt.\% & 25 & 1 \\
NMP in water, 80 wt.\% & 62 & 5 \\
Air & 62 & 0.5 \\
\hline
\end{tabular}

aspun with two orifice spinneret. waterflux of these membranes are almost equal and about $85 \mathrm{~L}-\mathrm{m}^{-2}-\mathrm{hr}^{-1}-\mathrm{bar}^{-1}$.

\section{Discussion}

In the systems investigated here no delay of demixing was found. This is due to the presence of PVP in the polymer solution. Delay times for a PES solution without PVP have been measured by Van 't Hof et al. [5]. They were strongly dependent on the nonsolvent used and could be very large, e.g. $1200 \mathrm{sec}$ for glycerol.

The velocity of loss in transmittance for the different nonsolvents as found by us has the same sequence as the delay times measured by Van ' $t$ Hof et al. [5]. This means that even though in all cases the polymer solution starts to demix immediately the demixing rate depends on the nonsolvent used.

For gas-separation membranes a certain contact time with the first coagulation medium (with finite delay time) is necessary in order to sufficiently increase the polymer concentration in the toplayer. In the systems discussed here the polymer solutions when brought in contact with the first coagulation medium demix instantaneously and thus pore formation starts immediately. In the second bath the strong coagulant water is used and the structure in the toplayer is fixed rapidly hindering further growth of pore sizes. From this it is clear 
that to obtain small pores in the toplayer which will be generated in the first coagulation medium, formation and growth of pores should be slow or has to be stopped rapidly. So for a thin, selective toplayer either demixing has to be slow or the contact time with the first coagulation medium must be very short.

Below the toplayer demixing will start when the fiber has entered the waterbath. In the sublayer the length of the diffusion path between demixing front and coagulation bath is larger and the densified toplayer will form a barrier slowing down the flux of solvent and nonsolvent. Thus compared to the interface more solvent is present. Also in the sublayer the polymer concentration is lower as it has not been increased in the first coagulation medium. Because of the presence of PVP, in the sublayer instantaneous demixing takes place according to the spinodal mechanism as proposed by Boom [3]. According to the theory of spinodal demixing the relatively high solvent concentration in the sublayer will lead to a structure consisting of large and interconnected pores.

In the experiments shown in Table 1 the residence time in the first liquid layer was $0.5 \mathrm{sec}$ in all cases. For glycerol with a slow demixing rate a high flux, high retention membrane would be expected. However the best results are found for 1-pentanol as the first coagulant. This discrepancy is due to a different effective contact time with the first coagulation medium. In all cases the polymer solution enters the second bath after $0.5 \mathrm{sec}$. In the case of glycerol mixing with water is slow due to the high viscosity of glycerol (945 cPoise) and glycerol remains in contact with the fiber for a significant time. The effective residence time is therefore much longer (about $30 \mathrm{sec}$ ). On the contrary pentanol is only slightly miscible with water and has a lower density than water. As soon as the polymer solution enters the waterbath pentanol is removed from the surface and floats on top of the waterbath. The effective residence time of pentanol is therefore little more than $0.5 \mathrm{sec}$.

Compared to the conventional spinneret the triple layer spinneret can lead to membranes with better performances. But as shown here, the type of the first coagulation medium and the contact time with this liquid layer are very important parameters and have to be investigated more thoroughly.

As said before the structure of the toplayer has to be fixed rapidly in the second bath. Water is a strong coagulant and therefore very suitable for this purpose. If the temperature of the waterbath is increased membranes are obtained with lower retentions while the waterflux is almost constant. This indicates that at higher temperatures more coalescence of the nuclei takes place, resulting in larger pores. Also from Table 3 it is clear that pore size is larger when a waterbath of higher temperature is used.

With this triple layer spinneret it is also possible to obtain microporous toplayers as shown in Table 3. The high solvent content in the first coagulation medium causes a low outdiffusion of solvent. Therefore the polymer concentration in the toplayer will be low. As was mentioned before a low polymer concentration results in a structure with large pores. For ultrafiltration membranes with the selective toplayer at the bore side of the fibers the new spinneret has no positive influence on flux or retention. However there are some other advantages of using a liquid layer over that of an airgap. A liquid bath is not as much influenced by environmental conditions as an airgap. Secondly in the case of an airgap the temperature of the waterbath has to be far above room temperature to obtain pore sizes in the micrometer range. From these results the triplet spinneret seems to be a promising tool for spinning microfiltration membranes but more research on this subject has to be done. 


\section{Conclusions}

Preliminary experiments indicate that the dual bath system using a triple layer spinneret is a suitable technique for the spinning of hollow fiber ultrafiltration membranes. The addition of PVP to the polymer solution is essential to induce demixing in the first coagulation medium and to obtain a porous toplayer. The selection of the type of liquid and the effective contact time of the polymer solution with the first coagulation medium are important parameters. Using 1-pentanol as the first external coagulation bath and water as the second bath high flux membranes can be prepared showing good retention for BSA. For membranes with the selective toplayer at the bore side of the fiber this technique can be used to produce pore sizes at the outer surface up to 5 $\mu \mathrm{m}$.

\section{References}

1 (a) L.E.S. Brink and D.J. Romijn, Reducing the protein fouling of polysulfone surfaces and polysulfone ultrafiltration membranes: Optimization of the type of presorbed layer, Desalination, 78 (1990) 209.

(b) G. Capannelli, A. Bottino, V. Gekas and G. Tragardh, Protein fouling behaviour of ultrafiltration membranes prepared with varying degrees of hydrophilicity, Proc. Biochem. Int., December (1990) 221.

(c) A.S. Jonsson and B. Jonsson, The influence of nonionic and ionic surfactants on hydrophobic and hydrophilic ultrafiltration membranes, J. Membrane Sci., 56 (1991) 49.

2 H.D.W. Roesink, Microfiltration, membrane development and module design, Thesis Univ. of Twente, The Netherlands, 1989.

3 R.M. Boom, Membrane formation by immersion precipitation: the role of a polymeric additive, Thesis Univ. of Twente, the Netherlands, 1992, (Parts will be submitted for publication in J. Membrane Sci.).

4 I. Cabasso, E. Klein and J.K. Smith, Polysulfone hollow fibers. II. Morphology, J. Appl. Polym. Sci., 21 (1977) 165.

5 J.A. van 't Hof, A.J. Reuvers, R.M. Boom, H.H.M. Rolevink and C.A. Smolders, Wet spinning of asymmetric gas separation membranes with high selectivity by a dual-bath coagulation method, J. Membrane Sci., 70 (1992) 17.

6 (a) G.H. Koops and S. Li, Het spinnen van asymmetrische holle vezelmembranen met een dicht niet-porcuze toplaag en een poreuze onderlaag, resp. met zowel een poreuze toplaag als een poreuze onderlaag, Dutch Patent 91.02151, 1991.

(b) S. Li, G.H. Koops, M.H.V. Mulder, Th v.d. Boomgaard and C.A. Smolders, Wet spinning of asymmetric hollow fibers with a new type of spinneret, to be published.

7 A.J. Reuvers and C.A. Smolders, Formation of membranes by means of immersion precipitation. Part II. The mechanism of formation of membranes prepared from the system CA/acetone/water, J. Membrane Sci., 34 (1987) 67. 